\title{
Primary Urothelium Carcinoma of the Distal Urethra in a Male: Case Report and Literature Review
}

This article was published in the following Dove Press journal: OncoTargets and Therapy

\author{
Ning Wang ${ }^{1,2}$ \\ Jingxian $\mathrm{Min}^{3}$ \\ Qiang $\mathrm{Wei}^{4}$ \\ Wanlong $\operatorname{Tan}^{4}$ \\ Qiang Dang ${ }^{4}$ \\ 'Department of Radiotherapy, Nan Fang \\ Hospital, Southern Medical University, \\ Guangzhou City, Guangdong Province \\ 510515, People's Republic of China; \\ ${ }^{2}$ Department of Oncology, Kanghua \\ Hospital, Dongguan City, Guangdong \\ Province 523000, People's Republic of \\ China; ${ }^{3}$ Department of Health \\ Management, Nan Fang Hospital, \\ Southern Medical University, Guangzhou \\ City, Guangdong Province 5105I5, \\ People's Republic of China; ${ }^{4}$ Department \\ of Urology, Nan Fang Hospital, Southern \\ Medical University, Guangzhou City, \\ Guangdong Province 510515, People's \\ Republic of China
}

\begin{abstract}
Male primary urethral urothelium carcinoma is a rare clinical case. Here, we detail a case of a 58-year-old man with primary urothelium carcinoma of the distal urethra treated in our hospital. The patient with a neoplasm inside the external urethral orifice for 2 years, which was previously diagnosed as condyloma acuminata, had received photodynamic therapy for 3 times, with initial symptoms of urinary stream bifurcation and dysuria. The exfoliative urine cytology showed negative. Cystoscopy showed a tumor growing around the distal urethra. Biopsy and immunohistochemistry revealed high-grade papillary urothelium carcinoma. The patient received partial urethrectomy, followed by urinary bladder irrigation chemotherapy with epirubicin postoperatively. The corpus spongiosum was invaded while the corpus cavernosa were not. Postoperative pathological examination showed high-grade invasive urothelium carcinoma. There is no evidence of tumor recurrence, metastasis or surgical complications during a 61-month follow-up period. Male primary urethral urothelium carcinoma is a rare clinical case with particular clinical and pathological characteristics. There are still no established treatment guidelines and should be studied further.
\end{abstract}

Keywords: primary urethral carcinoma, urothelium carcinoma, distal urethra, partial urethrectomy, photodynamic therapy

\section{Background}

Male primary urethral carcinoma rarely occurs, whose morbidity accounts for less than $1 \%$ of all malignancies. While urothelium carcinoma (UC) usually arises in the urothelium of the prostatic urethra, squamous cell carcinoma (SCC) commonly takes place in the distal urethra. Primary UC of the distal urethra, where typically is lined by squamous cell, is particularly rare. ${ }^{1}$ Due to the rarity of this disease, the aetiology of UC of the distal urethra is not fully demonstrated and there is still no established treatment guideline. In this report we present a patient with UC of the distal urethra who had an experience of 3 times of photodynamic therapy with the urethral neoplasm and literatures pertaining to this infrequent entity are reviewed.

\section{Case Reports}

A 58-year-old man was presented to our clinic in December 2014 with a 2-year history of urinary stream bifurcation and dysuria. In September of 2004, the patient noticed a small neoplasm near the external urethral orifice, which was removed by the patient himself without any physician visit. From December of 2012, as urinary stream
Wanlong Tan

Department of Urology, Nan Fang Hospital, Southern Medical University, Guangzhou

City, Guangdong Province 510515, People's

Republic of China

Email27064918I@qq.com 
bifurcation and dysuria occurred, a neoplasm was noted inside the external urethral orifice which was diagnosed as condyloma acuminata and treated with photodynamic therapy in the local hospital. However, it brought no resolution to his symptoms, while he developed into urinary frequency and increased nocturia about one year ago. In October 2014, the patient received another two times of photodynamic therapy for the urethral mass, but there was still no relief of symptoms.

After admission to our department, physical examination revealed a palpable mass, diameter of which was about $3 \mathrm{~cm}$, inside the external urethral meatus. No palpable inguinal lymph nodes were noted. The exfoliative urine cytology showed negative. Computed tomography (CT) showed a penile occupying lesion $(1.2 \mathrm{X} 1.1 \mathrm{X} 3.5 \mathrm{~cm} 3)$ close to the external orifice of urethra without any other lesion and swollen lymph nodes. Cystoscopy revealed a tumor growing around the urethra about $1 \mathrm{~cm}$ from the external urethral meatus and biopsy was taken (Figure 1A). Histopathologic examination reported urothelial hyperplasia and squamous cell differentiation arranged in papilla (Figure 2). Immunohistochemical analysis was carried out and reported as following: P16(-), P53(partly+), CK7(+), CK8(+), CK20(-), Uroplakin II (-), P63 $(+)$, Ki-67(+,50\%), confirming the diagnosis of high-grade papillary urothelium carcinoma (Figure 3). The patient received partial urethrectomy with urethral meatoplasty. The tumor appeared to invade the corpus spongiosum (Figure 1B). Thus, the corpus spongiosum was resected and corpus cavernosa were preserved. Postoperative pathological examination indicated high-grade invasive urothelium carcinoma, infiltrating the full-thickness urethral wall. Surgical margin was clear of tumor. The preoperative CT scan showed no lymph nodes in pelvic and groin. We also found no lymph nodes throughout the whole surgical process. The TNM staging was T2N0M0 according to TNM classification of the 8th version of AJCC.


Figure I (A) Cystoscopy image showing a neoplasm grown surrounding the distal urethra. (B) An ideograph displaying the appearance and invasion range of the tumor in the urethra. 

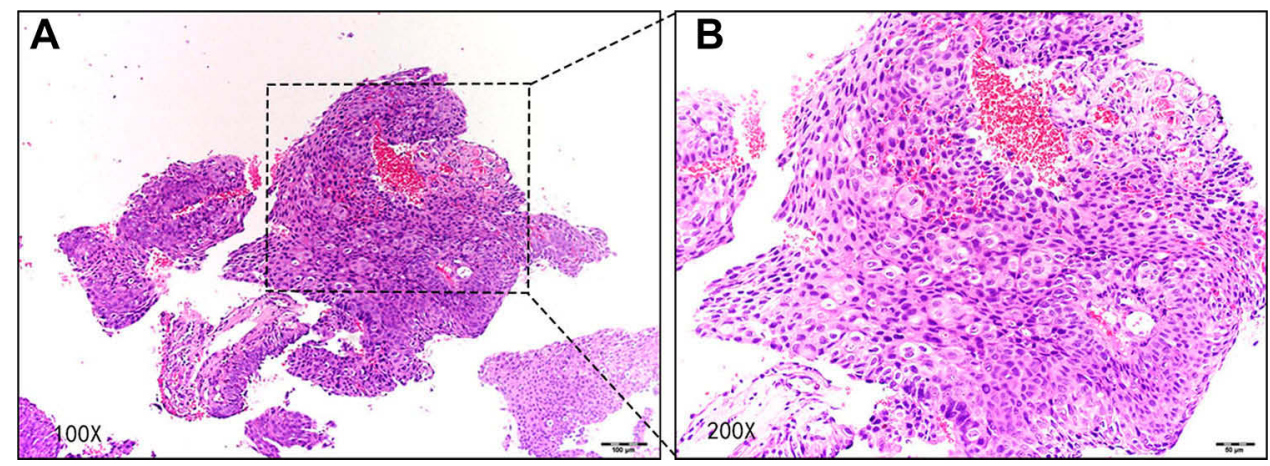

Figure 2 Microphotograph of the mass revealed high-grade urothelium carcinoma (hematoxylin-eosin, $\times 100$ magnification $(\mathbf{A})$ and $\times 200$ magnification $(\mathbf{B})$ )
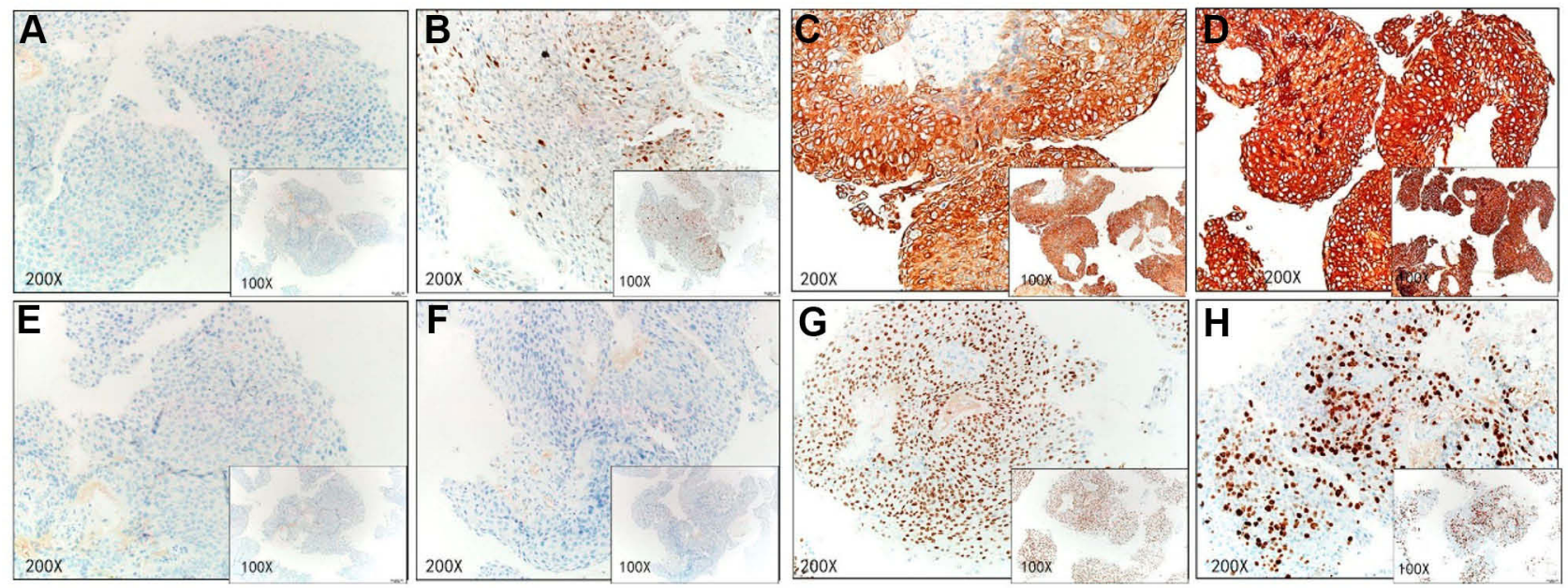

Figure 3 Immunohistochemistry of the tumor revealing: PI6(-) (A), P53(partly+) (B), CK7(+) (C), CK8(+) (D), CK20(-) (E), Uroplakin II (-) (F), P63(+) (G), Ki-67(+,50\%) (H).

The patient was treated with urinary bladder irrigation chemotherapy with epirubicin postoperatively. He made an uneventful postoperative recovery and was discharged on postoperative day 8 . The patient was under the follow-up periodically. In the first 2 years after surgery, patient was taken follow-up every 3 months. In the following 2-5 years after surgery, patient was taken follow-up every 6 months. After 5 years of the surgery, patient was taken follow-up every year. The follow-up strategy including physical examination, imaging examination (abdominal $\mathrm{CT}$, inguinal and pelvic $\mathrm{CT}$, chest X-ray), urinalysis, and cystoscope. After 61 months follow-up period, there is no evidence of tumor recurrence and metastasis. There is no evidence of surgical complications.

We confirm that written informed consent has been provided by the patient to have the case details and images published.

\section{Discussion}

Primary urethral carcinoma is a rare tumor. The histologic types of urethral carcinoma include UC, SCC and adenocarcinoma and others. It is well known that UC may show diverse histological differentiation into a wide spectrum of components, including squamous, glandular, small cell, micropapillary, sarcomatoid, and plasmacytoid subtypes. ${ }^{2}$ In the present case, UC in the distal urethra is extremely rare, as it is SCC that commonly occurs in this region, and this is the second report describing UC with squamous differentiation in the distal urethra. ${ }^{3}$

It is well recognized that tobacco smoking is a risk factor of UC of both bladder and upper tract, while carcinogenesis of upper urinary tract is also associated with occupational exposure to aromatic amines (benzidine and $\beta$-naphtylanine included) and ingestion of aristolochic acid in certain Chinese herbs. ${ }^{4}$ Nevertheless, risk factors for primary 
urethral UC are not clearly indicated. In a previous case report, human papillomavirus 16 was detected in transitional cell carcinoma of the navicular fossa, implying the possibility of the correlation between HPV infection and urethral UC. ${ }^{5}$ The patient in the present case denied related exposure, but he had a smoking history of 40 years, averagely 40 cigarettes per day ( smoking index $=1600$ ), and was once diagnosed with condyloma acuminata which was caused by HPV infection. Furthermore, the patient had received photodynamic therapy for the urethral neoplasm for three times in 2 years, which raised a question that whether frequent photodynamic therapy would induce oncogenesis. Several studies had reported UC following photodynamic therapy. There is a reason to suspect that the development of UC, at least partially, was related to repeated stimulation of photodynamic therapy for condyloma acuminata in this patient. The potential carcinogenic effect of photodynamic therapy requires further study and the clinicians' more careful follow-up to their patients.

Symptoms of primary urethral carcinomas are non-specific. At initial presentation, a history of visible hematuria or bloody urethral discharge is the most common $(62 \%$ of the cases). Further symptoms of locally advanced disease include an extraurethral mass (52\%), bladder outlet obstruction $(48 \%)$, pelvic pain $(33 \%)$, urethrocutaneous fistula (10\%), abscess formation (5\%), or dyspareunia. ${ }^{6}$ In our case, the patient was presented with urinary stream bifurcation and dysuria, features of urinary obstruction, with a palpable mass in the glans penis, but no hematuria history was noted. Given the non-specific nature of presenting symptoms, a series of examinations should be carried out to make a diagnosis timely. Physical examination should involve bilateral inguinal palpation to assess the presence of enlarged lymph nodes, as in primary urethral carcinoma, palpable adenopathy in most cases is caused by metastasis. ${ }^{7}$ Exfoliative urine cytology is helpful in diagnosis of urethral UC, with a sensitivity of $80 \%$ in terms of UC in male. ${ }^{8}$ Magnetic resonance imaging (MRI) or computed tomography (CT) is widely utilized to access the extent of local tumor, lymphatic and distant metastasis, especially metastasis of inguinal and pelvic lymph nodes. Urethrocystoscopy aims to identify the appearance and local extent of the tumor, followed by transurethral biopsy to confirm the diagnosis. In the case reported above, the histopathology examination showed UC with squamous differentiation. Considering the rareness of this entity in the male distal urethra, a series of Immunohistochemical detection was performed to make a final diagnosis. Previous case reports had no information about the immunohistochemical feature of urethral UC. In our case, Immunohistochemistry of the specimen showed: P16(-), P53(partly+), CK7(+), CK8(+), CK20(-), Uroplakin II (-), P63(+), Ki-67(+,50\%), and had provided further support to make an accurate diagnosis.

The rare nature of primary urethral UC makes it difficult to establish a common treatment guideline. In the reviewed cases, partial or total penectomy is the major treatment option for high-grade invasive lesions, ${ }^{9}$ while transurethral resection or local excision forms the mainstray of therapy for low-grade noninvasive lesions. ${ }^{10}$ However, current outcome data is inadequate to highlight the advantage of one treatment over others because of the small number of male urethral UC cases reported over a long period with the use of different modalities of treatment. Dalbagni et $\mathrm{al}^{11}$ reviewed 46 men with primary carcinoma of the bulbar and anterior urethra, proposing that locally advanced male urethral cancer should be treated aggressively with chemoradiation and penectomy for tumors of the anterior urethra and cystoprostatectomy with penectomy for tumors of the posterior urethra, for surgical resection is inadequate as the sole modality of treatment. Smith et al $^{12}$ studied the medium-term data from patients with distal urethral carcinoma who were treated with penile-preserving surgery, presenting a mean (median, range) follow-up of $26(20.5,9-58)$ months and $78 \%$ of the patients were alive and free of disease as the study was completed, concluding that penile-preserving surgery is feasible for distal urethral cancers and provides excellent local control. The role of Bilateral inguinal lymphadenectomy is not clearly defined, especially that of prophylactic lymphadenectomy. It is reported that bilateral groin dissection carries a high risk of complications and associated morbidity, therefore lymphadenectomy is recommended in cases of enlarged nodes and suspected metastatic disease. ${ }^{13}$ In the present case, the patient was treated with partial urethrectomy, followed by urinary bladder irrigation chemotherapy by epirubicin postoperatively. The corpus cavernosa were preserved, and inguinal lymphadenectomy was not performed because there was no evidence of inguinal or pelvic lymphadenopathy in preoperative examination. There was no evidence of recurrence or metastasis during a 61-month follow-up period. Accordingly, we suggest that partial urethrectomy and adjuvant intraurethral chemotherapy possibly is an effective treatment modality for relatively earlier stage of invasive male primary urethral UC (T1-2N0M0). Inguinal lymphadenectomy is optional depending on the preoperative examination. 
In summary, we reported a case of male primary UC of the distal urethra with particular histological and immunohistochemical characteristics. Due to the rarity of this entity, a uniform treatment guideline has not been established, and the risk factors and treatment outcomes are not fully demonstrated. Regarding this patient, we raised the suspicion of repeated photodynamic therapy being one of the carcinogenic factors. The treatment outcome of the current patient supports the use of Partial urethrectomy and intraurethral chemotherapy as a means to control localized UC of the distal male urethra. Further studies need to be carried out to improve the outcomes of patients with this disease.

\section{Acknowledgments}

This work was supported by National Natural Science Foundation of China (No.81502577), and Outstanding Youths Development Scheme of Nanfang Hospital, Southern Medical University (No.2016J007).

\section{Disclosure}

Ning Wang and Jingxian Min shared co-first authorship. Wanlong Tan and Qiang Dang shared co-corresponding authorship. The authors declared that they have no conflicts of interest in this work. We declare that we do not have any commercial or associative interest that represents a conflict of interest in connection with the work. We have solicited the patient's agreement before writing this case report. We confirm that written informed consent has been provided by the patient to have the case details and images published. No institutional approval was required to publish the case details.

\section{References}

1. Resnick MJ, Wein AJ. Transitional cell carcinoma of the fossa navicularis in a man with preexisting adenocarcinoma of the prostate. Urol Int. 2006;76(2):186-188. doi:10.1159/000090887

2. Amin MB. Histological variants of urothelial carcinoma: diagnostic, therapeutic and prognostic implications. Mod Pathol. 2009;22(Suppl 2):S96-S118. doi:10.1038/modpathol.2009.26

3. Fernando JJ, Wanas TM. Primary transitional cell carcinoma of the anterior urethra: a rare presentation. Genitourin Med. 1991;67 (3):244-246. doi:10.1136/sti.67.3.244

4. Burger M, Catto JWF, Dalbagni G, et al. Epidemiology and risk factors of urothelial bladder cancer. Eur Urol. 2013;63(2):234-241. doi:10.1016/j.eururo.2012.07.033

5. Sumino Y, Emoto A, Satoh F, et al. Transitional cell carcinoma of the navicular fossa detected human papillomavirus 16. Int J Urol. 2006;13(5):645-647. doi:10.1111/j.1442-2042.2006.01378.x

6. Gheiler EL, Tefilli MV, Tiguert R, et al. Management of primary urethral cancer. Urology. 1998;52(3):487-493. doi:10.1016/S00904295(98)00199-X

7. Dayyani F, Hoffman K, Eifel P, et al. Management of advanced primary urethral carcinomas. BJU Int. 2014;114(1):25-31. doi:10.1111/bju. 12630

8. Touijer AK, Dalbagni G. Role of voided urine cytology in diagnosing primary urethral carcinoma. Urology. 2004;63(1):33-35. doi:10.1016/ j.urology.2003.08.007

9. Lalude AO, Forbes KA. Urethrographic appearance of extensive transitional cell carcinoma of the anterior urethra. Urol Radiol. 1984;6(3-4):221-222. doi:10.1007/BF02923730

10. Harty JI, Mojsejenko IK. Transitional cell carcinoma of the anterior urethra. J Surg Oncol. 1982;21(2):121-124. doi:10.1002/ jso. 2930210212

11. Dalbagni G, Zhang ZF, Lacombe L, Herr HW. Male urethral carcinoma: analysis of treatment outcome. Urology. 1999;53(6):11261132. doi:10.1016/S0090-4295(98)00659-1

12. Smith Y, Hadway P, Ahmed S, et al. Penile-preserving surgery for male distal urethral carcinoma. BJU Int. 2007;100(1):82-87. doi:10.1111/j.1464-410X.2007.06901.x

13. Hakenberg OW, Franke H-J, Froehner M, et al. The treatment of primary urethral carcinoma-the dilemmas of a rare condition: experience with partial urethrectomy and adjuvant chemotherapy. Oncol Res Treat. 2001;24(1):48-52. doi:10.1159/000050282
OncoTargets and Therapy

\section{Publish your work in this journal}

OncoTargets and Therapy is an international, peer-reviewed, open access journal focusing on the pathological basis of all cancers, potential targets for therapy and treatment protocols employed to improve the management of cancer patients. The journal also focuses on the impact of management programs and new therapeutic agents and protocols on patient perspectives such as quality of life, adherence and satisfaction. The manuscript management system is completely online and includes a very quick and fair peer-review system, which is all easy to use. Visit http://www.dovepress.com/ testimonials.php to read real quotes from published authors. 